\title{
Modeling Integrated Mosquito Management for Klang Port: Reducing the Risk of the Re-Introduction of Zika Virus intoMalaysia
}

\author{
Thomas M. Kollars, Jr. \\ Jr., College of Health Sciences, Liberty University, 1971 University Blvd, Lynchburg, VA 24515 \\ Previous address Brigade Surgeon, $415^{\text {th }}$ Chemical Brigade USAR
}

\begin{abstract}
The global expansion of Zika virus (ZIKAV) is of great concern to medical and public health officials. Although ZIKAV does not appear to be currently circulating in Malaysia, positive ZIKAV mosquitoes and people were detected in the past and the potential is there for reintroductionand epidemic. Identifying routes of entry into areas where suitable vectors are present and providing information concerning integrated mosquito management (IMM) are critical to the prevention and control of Zika virus and its vectors. Because of the important role maritime ports often play in the invasion of medically important mosquito species, the port of Klang, to the west of Kuala Lumpur, was evaluated for susceptibility of invasion by ZIKAV infected mosquitoes and to identify zones for IMM using the Bioagent Transport and Environmental Modeling System (BioTEMS). BioTEMS has been used for modeling and planning of defense against biological weapons of mass destruction and infectious disease, including ZIKAV in Brazil and Florida, USA.BioTEMS indicated an index of 100\% suitability for the invasion of ZIKAV through the port area and identified zones and surveillance sites for IMM. Medical personnel and public health officials in Malaysia should maintain arboviral and vector surveillance and control around Klang Port and other sea ports in Malaysia.Integrated mosquito management plans should be in place before ZIKAV arrives ashore.
\end{abstract}

Keywords:Aedes, vector, invasive species, martime ports, biological agents

\section{Introduction}

Zika (ZIKAV), dengue, and chikungunya viruses are spreading rapidly to new geographic areas on a global scale. Several medically important mosquito species are invading new geographic regions by exploiting niches that are similar to their natural home ranges and new haplotypes are being introduced into resident mosquito populations. Also of concern, is the lack of knowledge concerning arboviruses and mosquito prevention and control for invasive species.About 30 years ago, ZIKAV was circulating in SE Asia, and isolates of the virus were made from the Aedes aegypti mosquito in Malaysia. However, in a recent survey, a large proportion of respondents did not consider Malaysia at risk of ZIKAV and there was a lack of knowledge of how the virus is spread and what measures can be taken to prevent transmission (Khan et al. 2016). The principle factor responsible for the introduction of disease vectors into new geographic areas is by transport into air and maritime ports (Tatem et al. 2006). These invasive mosquitoes, and the pathogens they carry, may pose significant medical and veterinary health risks to naïve populations. BothAe aegyptiand Ae albopictus have been recorded in the Kuala Lumpur area (Roslan et al. 2013). Unfortunately insecticide resistance is also common in these two mosquito species in Malaysia, depending on the geographic population (Ishak et al. 2013).A container ship from the ZIKAV endemic ports in Brazil takes approximately 45 days at sea to reach Malaysia (Ports.com, 2016). Therefore, the possibility of introducing an infected haplotype of Ae aegypti or Ae albopictus exists. Because of the import role maritime ports often play in the invasion of medically important mosquito species, the Klang Port, located on the west coast,was evaluated for susceptibility of invasion by ZIKAV. In addition integrated mosquito management (IMM) zones and surveillance sites were identified to assist medical and public health officials in mitigating risk of ZIKAV.

\section{Materials and Methods}

ArcGIS geospatial analysis software, Statistica statistical software and the Bioagent Transport and Environmental Modeling System (BioTEMS) were used to analyze geographic information and conduct data analysis. BioTEMS utilizes up to several hundred abiotic and biotic factors to produce risk and vulnerability assessments for biological agents and infectious diseases. Examples of biotic and biotic factors include; pathogen strain, vector/host relationship, vectorial capacity, host/vector physiology, colonization ability, population dynamics of hosts and vectors, soil, shade, and weather conditions, such as wind, temperature, precipitation, shade. Analytical methods within BioTEMS includes; artificial intelligence, fuzzy logic, niche analysis, random forests and general additive regression and statistics. BioTEMS has been used in risk analysis 
and vulnerability assessment for biological weapons of mass destruction, including; Bacillus anthracis, Francisellatularensis, Yersinia pestis, and Crimean-Congo hemorrhagic fever virus. These assessments were used during consequence management planning for military facilities in the U.S. and internationally, for government facilities and during presidential/national national events. BioTEMS has also be used with HPAC to analyze bioagent information and to optimize placement of U.S. Army Biological Integrated Detection Systems. In addition to applications for biological weapons defense, BioTEMS has been used for infectious disease modeling and planning.

The BioTEMS TIGER model was developed to assist in identifying areas at highest risk for invasive mosquito species, associated pathogens and to optimize surveillance and control efforts (Kollars et. al. 2016). Within the BioTEMS TIGER model; Transport- identifies the point of origin, method and rate of transport to a locality, Introduction- area of initial introduction/invasion and preliminary spread of species or immigrant haplotypes into a locality, Gap- determines the area where vector/pathogen initially spreads once it has gained a foothold, Escalade- incorporates abiotic and biotic resistance to invasion, and Residence and recruitment incorporates factors and areas where vector/associated pathogens add to genetic diversity or become endemic and recruits con-specifics. The BioTEMS model has been used to identify areas at risk for ZIKAV and identify areas for integrated mosquito management in maritime ports in Miami, Tampa, and Mayport Naval Port in Florida (Kollars and Kollars 2016, Kollars 2017). Areas at risk of Zika virus and IMM zones and surveillance sites were identified based on the BioTEMS TIGER model should Zika virus be introduced through the Klang Port. BioTEMS and ArcView were used to produce output into Google Earth.

\section{Results and Discussion}

The BioTEMS TIGER identified an index value of $100 \%$ for the Klang Port as suitable for the invasion and establishment of ZIKAV infected Aedes spp. and haplotypes. The area of risk for Introduction/Invasion by ZIKAV infected Aedesspeciesand haplotypes is approximately $85 \mathrm{~km}^{2}$ in area (Figure 1). This area stretches eastward from the Klang Port to SalatKlang Highway. Public health officials can use the output to implement mosquito control, reduce breeding habitats, and conduct epidemiologic surveillance of arbovirus infection in mosquitoes and humans. The Gap Infiltration Zone is contained in an area approximately $220 \mathrm{~km}^{2}$. Mosquito surveillance and arbovirus epidemiologic surveillance of mosquitoes and humans should be conducted in the Gap Infiltration Zone. Should ZIKAV be detected in the Introduction/Invasion Zone, mosquito control using pesticides and other IMM measures should immediately be conducted in the Gap Zone.Recommended surveillance sites were identified throughout the area to assist in surveillance. BioTEMS predicted that if ZIKAV was imported through the Klang Port, it could spread through the mosquito population, endangering the human population and increasing the probability of ZIKAV becoming established. The output from the BioTEMS TIGER model can be used to open the door to a discussion and to educate the public on ZIKAV prevention and control. Interventions should be customized to improve the knowledge and attitudes of the public towards ZIKAV prevention and control (Khan et al. 2016). The presence of possible vectors of ZIKAV varies geographically in Malaysia affecting the IMM plan. The public also must be made aware of the vector species so they may take the best methods to protect themselves and their families. For example, in some areas of nearby Kuala Lumpur, Ae albopictus appears to be the primary vector of dengue virus and not Ae aegypti, the normal suspect (Wan-Norafikah et al. 2009). Therefore, arbovirus surveillance and control should include both Ae aegypti and Ae albopictus within the IMM plan.

Another critical factor in IMM and control of Aedesmosquitoes is choosing the proper pesticide and optimally applying the pesticide to maximize effect. Investigating the periods of activity and distribution of local Aedesmosquito populations is necessary if control is to be obtained. Relying solely on aerial spray of naled from aircraft to control adult Aedesmosquitoes may meet with failure, in addition naled is only effective for a few hours in areas that have much cover. Populations may rebound in a week or soafter using Naled (Sutherland et al. 1978; Howard and Oliver, 1997). Other pesticides, such as malathion, can be used, however pesticide resistance must be monitored. Using backpack sprayers and truck mounted application of pesticides, in addition to aerial spraying from aircraft, should be a part of the IMM strategy for controlling adult Aedes mosquitoes. For larval control, Bacillus thuringiensis israelensis (Bti) can be used to reduce Aedesmosquito populations, being applied by air craft, back pack sprayers, ULV truck mounted spraying, and by hand placement of granules or briquettes. In addition to Bti, insect growth regulators (IGR) such as methoprene can be utilized in sprays.

Entobac pesticide, with Bti as its active ingredient, has been used for the first time within a bait and used in spray or ProVector®applicators to kill both adult and larvae mosquitoes. The ProVector applicators with Entobac ${ }^{\mathrm{TM}}$ are placed inports, hotels, houses and mosquito breeding sites to control both adult and larval populations (Kollars et al. 2016). This is the first Bti based pesticide found to be effective in the field against both adult and larval mosquitoes (Yalwala et al. 2016). The technology used in the ProVector allows for the adult mosquito to ingest the pesticide and then transport the pesticide to larval breeding sites. In approximately 
7 days the adult mosquito dies and if the adult mosquito lands in water, the larval mosquitoes die as well. The ProVector with Entobac may provide a useful tool in killing larva mosquitoes in various breeding sites, including fire buckets containing water and waste tires. The ProVector tube can be hung in trees or on structures in and around maritime ports as well as on ships to help reduce the risk of ZIKAV infected mosquitoes from being imported into the Klang Port. In the event an infected traveler infects local mosquitoes, the presence of ProVectorapplicators with Entobac, or other long lasting environmentally safe pesticides, can reduce the mosquito population and reduce the risk of ZIKAV spreading.

In conclusion, as history indicates, both the human and mosquito population in Malaysiaare vulnerable to ZIKAV (Marchette et al. 1969, Khan et al. 2016). Dengue virus is transovarially transmitted in Aeaegypti and Aealbopictus in Malaysia (Rohani et al. 2014). The establishment of ZIKAV into new geographic areas could be facilitated if ZIKAV is transovarially or sexually transmitted by Aedes vectors; additional research in intraspecies transmission is needed. Identifying areas susceptible to introduction and invasion of infected mosquitoes and humans is critical in preventing the re-establishment of ZIKAV in Malaysia. Application of the correct pesticides, at alternating schedules with targeting the different mosquito stages, is essential to mitigate resistance issues and/or environmental contamination. Focusing control efforts primarily on travelers and not including ports of entry may do a disservice to the population to whom public health officials are protect. Medical personnel and public health officials should maintain arboviral and vector surveillance and consider increasing IMM around sea ports in Malaysia. Integrated mosquito management plans should be put into place before ZIKAV arrives ashore through infected mosquitoes or infected humans. Sam et al. (2016) posed a challenge for medical and public health professionals in Malaysia that is applicable worldwide, stating, "Innovative new methods for surveillance and control of vectors are needed to target mosquito-borne diseases as a whole."The recent application of BioTEMS beyond biological weapons of mass destruction defense to planning mitigation of ZIKAV and other infectious diseases, can assist public health officials in Malaysia and other countries susceptible to ZIKAV and other vector-borne diseases.

\section{Acknowledgement and Disclosure}

The views expressed in this publication are those of the authors and do not reflect the official policy of the United States Army or United States Government.

\section{References}

[1]. Howard JJ and Oliver J. (1997). Impact of naled (Dibrom 14) on the mosquito vectors of eastern equineencephalitis virus. J Am Mosq Control Assoc. 13:315-25.

[2]. Kollars, TM Jr. (2017) Assessing likely invasion sites of Zika virus infected mosquitoes incivilian and naval maritime ports in Florida. Res Rep Trop Med. 8:1-6.

[3]. Kollars, TM Jr, Kollars PG, and Hulsey B (2016). Reducing the Risk to Marine Ports fromInvasive Mosquito Species, Zika, Dengue, Chikungunya viruses and Filariasis. Intl J Med. 4:70-73.

[4]. Kollars TM Jr, JW Kollars. 2016. The Invasion of Zika Virus into Rio De Janeiro and Fortaleza, Brazil, Inside Out or Outside In? Ann. Com. Med. Prac. 2: 1015-1018.

[5]. Kollars TM. 2017. Assessing likely invasion sites of Zika virus infected mosquitoes and naval maritime ports in Florida. Res Rep Trop Med. 8:1-6.

[6]. Ishak IH, Jaal Z, Ranson H, Wondji CS (2015). Contrasting patterns of insecticide resistance andknockdown resistance (kdr) in the dengue vectors Aedes aegypti and Aedes albopictus from Malaysia Parasites \& Vectors 8:181, DOI 10.1186/s13071-015-0797-2.

[7]. Khan MU, Ahmad A, Arief M, Saleem F, Hassali MA, and Bhagavathula AS (2016). Publicknowledge and attitudes towards Zika virus and its prevention in Malaysia. Value in Health 19:423.

[8]. Sam JIC, Chan YF, Vythilingam I, Wan Sulaiman WY (2016). Zika virus and its potential re-emergence in Malaysia. Med J Malaysia 71:66-68.

[9]. Marchette NJ, Garcia R, and Rudnick A. (1969). Isolation of Zika Virus from Aedes aegyptiMosquitoes in Malaysia Am J Trop Med Hyg.18:411-415.

[10]. Muhammad-Aidil R, Imelda A, Jefffery J, Ngui R, Wan Yussoff WS, Aziz S, Lim YAL and Rohela M (2015). Distribution of mosquito larvae in various breeding sites in Nataional Zoo Malaysia. Trop Biomed 32: 183-186.

[11]. Ports.com (2016). http://ports.com/sea-route/port-of-klang,malaysia/port-of-santos,brazilRohani A, Azahary AA; Malinda M,Zurainee MN,,Rozilawati H (2014). Eco-virological surveyofAedes mosquito larvae in selected dengue outbreak areas in Malaysia. JVector Borne Dis. 51:327-332.

[12]. RoslanMA,ShafieA,Ngui R; Lim YL,Sulaiman W, Wan Y (2013). Vertical infestation of thedengue vectors Aedes aegypti and Aedes albopictus in apartments in Kuala Lumpur, Malaysia.J Am MosqCont Assoc. 29:328-36.

[13]. Sutherland DJ, Kent R and Downing J (1978). The effect of aerial ulvadulticiding with malathionandnaled on field populations of Aedes sollicitans. Mosq News. 38:488-491.

[14]. Tatem AJ, SI Hay SI, Rogers DJ (2006). Global traffic and disease vectors. Proc Natl AcadSciUSA. 103: 6242-6247.

[15]. Wan-Norafikah O, Chen CD, Soh HN, Lee HL, Nazni WA, and Sofian-Azirun M (2009). Surveillance of Aedes mosquitoes in a university campus in Kuala Lumpur, Malaysia. Trop Biomed. 26:206-15.

[16]. Yalwala S, JW Kollars JW, Chrisostim JW, Kasembeli G, Senessie C, Kollars PG, Kollars, TMJr (2016). Preliminary report on the reduction of adult mosquitoes in housing compounds in western Kenya using the ProVector Flower and Entobac bait pads containing Bacillus thuringiensis israelensis with honey bait. J Med Ent. 53:1242-1244. 
Figure 1. Area surrounding Klang Port at high risk of Zika virus (red) and recommended integrated mosquito management zone (yellow) should Zika virus be introduced through the port. Recommended areas for mosquito surveillance (blue circles)

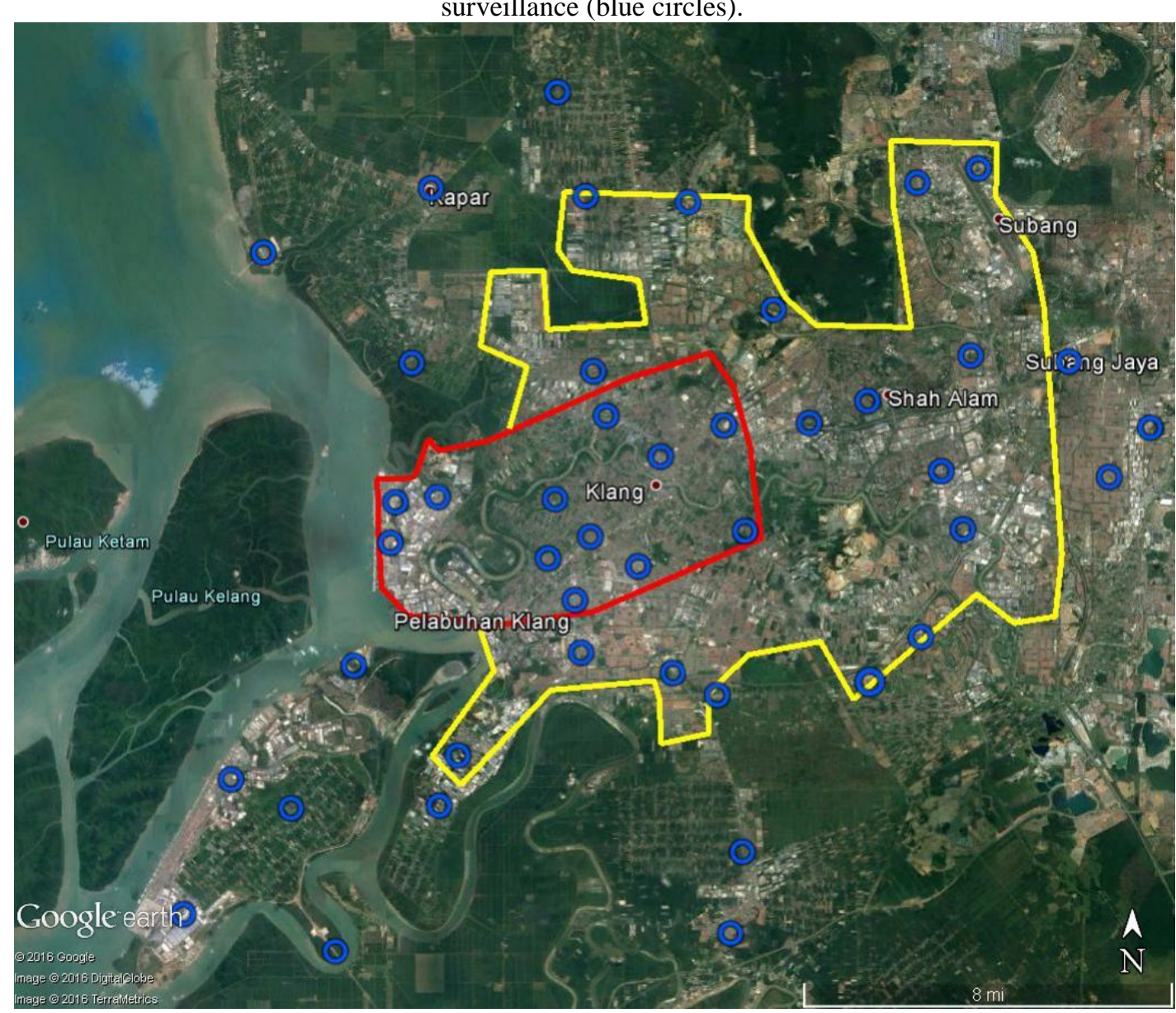

